\title{
Exercise Limitations by the Oxygen Delivery and Utilization Systems in Aging and Disease: Coordinated Adaptation and Deadaptation of the Lung-Heart Muscle Axis - A Mini-Review
}

\author{
Martin Burtscher \\ Department of Sport Sciences, Medical Section, University of Innsbruck, Innsbruck, Austria
}

\author{
Key Words \\ Oxygen delivery $\cdot$ Oxygen utilization $\cdot$ Heart $\cdot$ Lung $\cdot$ \\ Muscle $\cdot$ Aging $\cdot$ Disease $\cdot$ Exercise training $\cdot$ Adaptation • \\ Deadaptation
}

\begin{abstract}
Cardiorespiratory fitness (aerobic exercise capacity) is one of the most important prerequisites for successful aging in human beings and depends on adequate oxygen transport by the respiratory and circulatory systems from environmental air to the working muscles and the efficient utilization of oxygen by the mitochondria. A linear dose-response relation between aerobic exercise capacity, morbidity, mortality, and quality of life is well documented. The process of normal aging is associated with a variable reduction in functional capacity of the main organs involved in oxygen delivery and utilization. Integrated changes of the heart-lung muscle axis are termed here 'coordinated deadaptation', e.g. due to aging and disease, in contrast to the beneficial effects of 'coordinated adaptation', e.g. resulting from exercise training. Physical inactivity in aging persons initiates a circulus vitiosus resulting in coordinated deadaptation of the oxygen delivery and utilization systems mainly affecting the heartmuscle axis. Whereas in the healthy elderly the deadaptation
\end{abstract}

process starts from inactive locomotor muscles, the lung or the heart represent the origin in patients suffering from respiratory or cardiovascular diseases. Specific exercise training programs, considering the state of cardiorespiratory health and physical activity, are the most important and almost the only effective intervention to avoid or to break the circulus vitiosus, thereby promoting quality and expectancy of life in aging humans.

Copyright $\odot 2012$ S. Karger AG, Basel

\section{Introduction}

To maintain cardiorespiratory fitness is one of the most important prerequisites for successful aging in human beings. Cardiorespiratory fitness depends on adequate oxygen transport by the respiratory and circulatory systems from environmental air to the working muscles and the efficient utilization of oxygen by the mitochondria (fig. 1). Many studies have assessed individual fitness level as an objective measure of physical activity, consistently showing a strong dose-response relation between aerobic exercise capacity, morbidity, mortality, and quality of life [1]. Generally, the reduction of the mortality risk of sedentary subjects ranges between 10 and $25 \%$ for each

\section{KARGER}

E-Mail karger@karger.com

www.karger.com/ger
(C) 2012 S. Karger AG, Basel

0304-324X/13/0594-0289\$38.00/0
Prof. Martin Burtscher, MD, PhD

Department of Sport Sciences

Medical Section, University of Innsbruck

Fürstenweg 185, AT-6020 Innsbruck (Austria)

E-Mail martin.burtscher@uibk.ac.at 


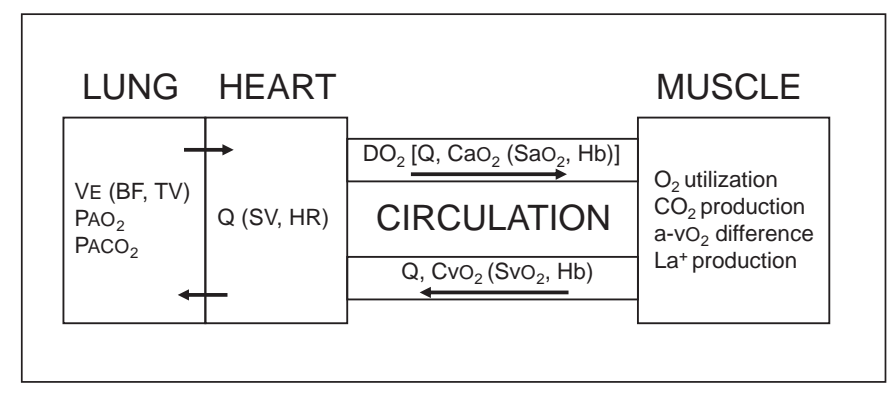

Fig. 1. Oxygen delivery and utilization systems. $\mathrm{VE}=$ minute ventilation; $\mathrm{BF}=$ breathing frequency; $\mathrm{TV}=$ tidal volume; $\mathrm{PAO}_{2}=$ alveolar oxygen partial pressure; $\mathrm{PACO}_{2}=$ alveolar partial pressure of carbon dioxide; $\mathrm{Q}=$ cardiac output; $\mathrm{SV}=$ stroke volume; $\mathrm{HR}=$ heart rate; $\mathrm{DO}_{2}=$ oxygen delivery; $\mathrm{CaO}_{2}=$ arterial oxygen content; $\mathrm{SaO}_{2}=$ arterial oxygen saturation; $\mathrm{Hb}=$ haemoglobin; $\mathrm{CvO}_{2}=$ mixed venous oxygen content $\mathrm{SvO}_{2}=$ mixed venous oxygen saturation; $\mathrm{a}-\mathrm{vO}_{2}$ difference $=$ arterio-venous oxygen difference; $\mathrm{La}^{+}=$lactate.

metabolic equivalent $\left(1 \mathrm{MET}=\right.$ approx. $3.5 \mathrm{ml} \mathrm{O}_{2} \cdot \mathrm{min}^{-1}$. $\mathrm{kg}^{-1}$ ) increase in aerobic exercise capacity up to approximately 13 METs [1]. Although aerobic exercise capacity inevitably decreases with aging, oxygen uptake at the anaerobic threshold remains about 3.5-fold higher in elite endurance athletes up to $70+$ years when compared to their untrained peers [2]. On the other hand, physical inactivity and disease may accelerate the decline of oxygen delivery and utilization capacities and the related exercise performance below the level necessary for performing daily activities. The process of normal aging is associated with a variable reduction in functional capacity of the main organs (lung, heart, skeletal muscles) involved in oxygen delivery and utilization. Several studies, including the Baltimore Longitudinal Study of Aging, have demonstrated that modifications occur in both structure and function of organs. For instance, skeletal muscles not regularly used will undergo hypotrophy and lose oxidative capacity. According to the concept of symmorphosis, functional capacities (and structure) of the cardiovascular and respiratory systems, responsible for oxygen delivery, will also decline when mitochondrial volume decreases [3]. Integrated changes of the heart-lung muscle axis are termed here 'coordinated deadaptation,' e.g. due to aging and disease, in contrast to the beneficial effects of 'coordinated adaptation', e.g. resulting from exercise training. Although there is a plethora of studies demonstrating the loss of aerobic exercise capacity with aging in health or disease and its improvement by exercise training, only little information is available on stimulus-spe- cific mechanisms of coordinated deadaptation and adaptation of the oxygen delivery and utilization systems. However, the understanding of such mechanisms may be of utmost importance to avoid detrimental effects of deadaptation and optimize the application of exercise training.

\section{The Oxygen Transport System}

Like other tissues, skeletal muscles rely on a continuous supply of oxygen at a rate which is precisely matched to changing metabolic requirements. These requirements increase steeply with increasing intensity of muscle work. If oxygen supply is inadequate, anaerobic metabolism compensates transiently for the lack of oxygen, resulting in the production of lactate. The transport of the environmental oxygen to the mitochondria of the skeletal muscles occurs as a series of steps: oxygen uptake in the lungs and oxygen delivery from the lungs to the mitochondria driven by the pumping heart. Even in a healthy aging organism, this system works energy efficiently by properly matching oxygen delivery with the metabolic demand. Adequate ventilation of the alveoli is necessary to maintain alveolar oxygen pressure $\left(\mathrm{PAO}_{2}\right)$ and to remove carbon dioxide $\left(\mathrm{CO}_{2}\right)$. $\mathrm{PAO}_{2}$ constitutes the driving pressure for the diffusion of oxygen into the pulmonary capillary blood. Oxygen is then transported from the lung to the tissues by the central circulation (fig. 1). Since oxygen is predominantly carried by the hemoglobin, the amount of oxygen that can be transported by the blood depends on the hemoglobin concentration of the blood. Oxygen delivery $\left(\mathrm{DO}_{2}\right)$ is determined by the cardiac output $(\mathrm{Q})$, the hemoglobin concentration $(\mathrm{Hb})$ and the level of its saturation with oxygen $\left(\mathrm{SaO}_{2}\right): \mathrm{DO}_{2}=\mathrm{Q} \times \mathrm{Hb} \times \mathrm{SaO}_{2} \times \mathrm{K}$, with $\mathrm{K}$ representing the coefficient for hemoglobin-oxygen binding capacity: $1.33 \mathrm{ml} / \mathrm{g}$. The amount of oxygen used by the mitochondria is given by the difference between the arterial and venous oxygen content $\left(\mathrm{CaO}_{2}-\right.$ $\mathrm{CvO}_{2} ; \mathrm{a}-\mathrm{vO}_{2}$ difference) indicating the amount of oxygen extraction (fig. 1). As the demand for oxygen increases during intense exercise or supply is diminished, oxygen extraction increases. According to the Fick principle, $\mathrm{VO}_{2}=\mathrm{Q} \times$ a- $\mathrm{VO}_{2}$ difference. $\mathrm{VO}_{2 \max }$ or aerobic capacity is defined as the highest achievable rate at which oxygen can be transported from air to tissues and utilized by the mitochondria during high-intensity exercise. The term 'maximum oxygen uptake' was introduced by Hill and Lupton [4] in 1923, who stated that there is an upper limit to oxygen uptake depending on the ability of the car- 
diorespiratory system to transport oxygen to the exercising muscles. Their $\mathrm{VO}_{2}$ max paradigm was mainly based on the observation that there is an upper limit (plateau) of oxygen uptake which cannot be forced to increase despite increasing the exercise intensity. This view has been questioned by some exercise physiologists because a large proportion of subjects (approx. 50\%) do not demonstrate a $\mathrm{VO}_{2}$ plateau despite exercising at maximal effort. The failure to reach a plateau has been interpreted as skeletal muscles being the limiting factor to $\mathrm{VO}_{2}$ max than the maximal $\mathrm{DO}_{2}$. However, many persons, especially the elderly, likely fatigue just when reaching $\mathrm{VO}_{2}$ max and consequently a plateau will not be apparent. At any rate, $\mathrm{VO}_{2 \text { max }}$ is considered as the result of integrated functioning of the lung-heart muscle axis at maximal exercise and ranges from below $20 \mathrm{ml} / \mathrm{min} / \mathrm{kg}$ in the very elderly subjects and in those with cardiovascular and/or lung diseases up to values of $90 \mathrm{ml} / \mathrm{min} / \mathrm{kg}$ in elite endurance athletes [5]. As proposed by the concept of coordinated (de) adaptation, a single disturbance in this multistep pathway is assumed to trigger adaptation or deadaptation in the remaining steps [6]. Aging, disease, and physical activity mostly affect various steps (organs) differently and the resulting adaptations or deadaptations will largely depend on the type, duration, intensity, and interaction of primary and secondary stimuli.

\section{Determinants of $\mathrm{Vo}_{2}$ max Related to Aging and Training}

\section{The Respiratory System}

At the exercise intensity equivalent to $\mathrm{VO}_{2}$ of $1 \mathrm{l} / \mathrm{min}$, pulmonary ventilation (VE) of about $25 \mathrm{l} / \mathrm{min}$ is necessary to adequately remove $\mathrm{CO}_{2}$ and to maintain $\mathrm{PAO}_{2}$ in healthy subjects. Thus, at a $\mathrm{VO}_{2}$ of $4 \mathrm{l} / \mathrm{min}$, VE must increase to $100 \mathrm{l} / \mathrm{min}$. If VE has additionally to compensate for lactate acidosis, it will increase to or above $120 \mathrm{l} / \mathrm{min}$. Despite this high VE demand, the individual maximal voluntary ventilation (MVV) usually far exceeds $\mathrm{VE}_{\text {max }}$ in healthy young individuals. In some highly trained young and older endurance athletes, however, the respiratory system may in fact become limiting. Exercise-induced arterial oxygen desaturation ( $>5 \%$ from resting levels of $98 \%$ ) in athletes may occur due to a shortened pulmonary capillary transit time at an extraordinarily high $\mathrm{Q}$ or due to an insufficient hyperventilatory response to exercise resulting in reduced $\mathrm{PAO}_{2}$ and consequently $\mathrm{SaO}_{2}$, or because of the rightward shift of the oxyhemoglobin dissociation curve due to lactic acidosis and hyperthermia [7]. In aging individuals, respiratory function progressively declines along with structural and functional changes, e.g. increasing rigidity of the chest wall, decline in respiratory muscle strength and endurance, loss of elastic recoil, decrease in the alveolar surface area, and a reduction in the number of capillaries perfusing the lung [8]. These agerelated changes might theoretically suggest that the respiratory system would progressively become limiting to $\mathrm{VO}_{2 \text { max }}$. However, the findings of Habedank et al. [9] do not support this assumption. The authors demonstrated a breathing reserve $[\mathrm{BR}=(\mathrm{MVV}-\mathrm{VE} / \mathrm{MVV}) \times 100]$ of $41 \%$ independent of age and gender in a relatively large sample of young and older healthy subjects. In addition, exercise-induced arterial hypoxemia is rare in the elderly population, but seems to be somewhat more prevalent in highly fit older subjects [10], similar to what is reported for highly trained young athletes [7]. The alveolar-to-arterial oxygen difference starts to widen at about $50 \%$ of $\mathrm{VO}_{2 \text { max }}$ and is about threefold higher at maximal exercise compared to rest, but is very similar in young and elderly subjects with average fitness [8]. The $\mathrm{VO}_{2 \text { max }}$ declines at a rate of approximately $10 \%$ per decade, but MVV (calculated from normal values of forced expiratory volumes in $\left.1 \mathrm{~s} ; \mathrm{FEV}_{1}\right)$ decreases at a rate of approximately $6 \%$ and lung diffusion capacity for carbon monoxide only at a rate of approximately $5 \%$ per decade, indicating no significant limit to $\mathrm{VO}_{2}$ max [8]. Whereas lung function has been shown not to be different between young trained and sedentary subjects, the age-related decline may be attenuated in well-trained elders compared to their sedentary peers. In conclusion, $\mathrm{VO}_{2}$ max is usually not limited by insufficient ventilation and pulmonary gas exchange in the healthy elderly, likely due to the fact that age-related reduction in the maximal metabolic demand of exercise occurs at a rate equal to or greater than the changes in the respiratory system. Effects of coordinated adaptation or deadaptation due to physical activity or inactivity will not largely affect the healthy respiratory system.

\section{The Heart, $\mathbf{Q}_{\max }$ and $\mathrm{Vo}_{2 \max }$}

The limiting role of the maximal $\mathrm{DO}_{2}$, particularly of the $\mathrm{Q}_{\max }$, is supported by the fact that $\mathrm{VO}_{2}$ max is typically achieved by exercise involving only about half of the total body muscles. Since the 1990s, it has been widely accepted that although there is no single limiting factor, $\mathrm{VO}_{2}$ max in healthy subjects performing maximal exercise testing at low altitude is predominantly limited by $\mathrm{Q}_{\max }$. When a large proportion of blood flow is directed to a rather 


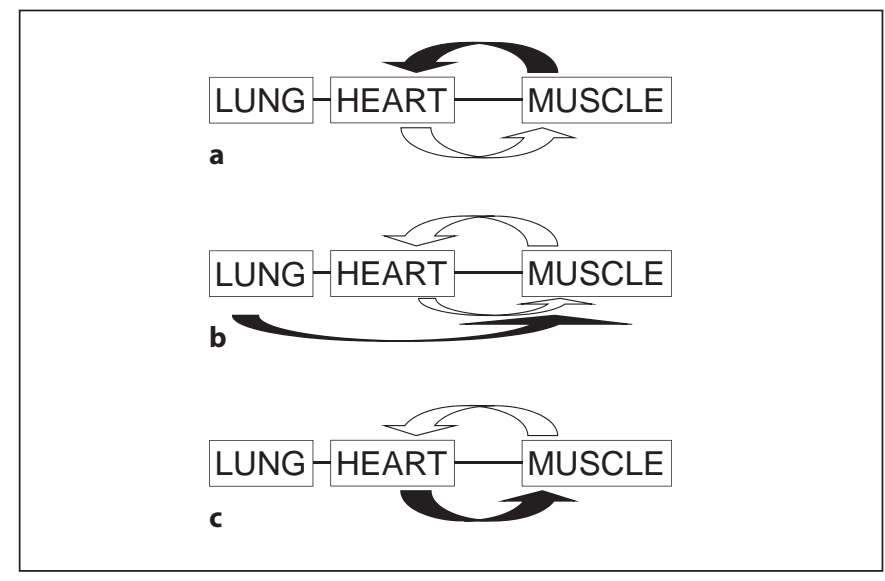

Fig. 2. Suggested feedback loops resulting from exercise training or physical inactivity in healthy individuals (a), chronic obstructive pulmonary disease (b), and chronic heart failure (c). The filled black arrows indicate the initial stimulus.

small group of muscles exercising maximally, $\mathrm{VO}_{2}$ is $2-3$ times higher as compared to the same muscles during whole body exercise [11]. These results convincingly demonstrate the significance of $\mathrm{Q}_{\max }$ (and maximal $\mathrm{DO}_{2}$ ) for $\mathrm{VO}_{2 \text { max }}$. It has been suggested that $70-85 \%$ of the limitation in $\mathrm{VO}_{2 \text { max }}$ is linked to $\mathrm{Q}_{\max }$. Ogawa et al. [12] demonstrated an approximate $72 \%$ contribution of $\mathrm{Q}_{\max }$ to $\mathrm{VO}_{2 \text { max }}$ changes in sedentary young and older subjects of both sexes, and an $81-89 \% \mathrm{Q}_{\max }$ contribution in trained young and older subjects of both sexes. Thus, it is not surprising that a decline of $\mathrm{Q}_{\max }$ with aging or in patients suffering from cardiac diseases will result in a dramatic decrease of $\mathrm{VO}_{2}$ max which, however, may be partly compensated by increasing oxygen extraction [13]. Whereas the reduction in $\mathrm{Q}_{\max }$ is associated with a decline in maximal heart rate, the higher $\mathrm{Q}_{\max }$ in trained older subjects is explained by larger stroke volumes (SV) $[12,14]$. Heart rate decreases (beats per minute) can be predicted according to the formula: $208-0.7 \times$ age, and the underlying mechanisms seem to be related to the decrease in intrinsic heart rate [15]. $\mathrm{VO}_{2}$ max gradually declines with aging in sedentary as well as in highly trained athletes. In sedentary adults of both sexes it decreases by approximately $10 \%$ per decade after the age of 30 years [14]. Trained individuals show a somewhat steeper decline in $\mathrm{VO}_{2 \text { max }}$, especially after the age of 50 years, but they have much higher mean levels at any age compared to sedentary persons $[2,14]$. Pronounced improvements in aerobic capacity are observed after endurance training in healthy young and older subjects (approx. 70 years). $\mathrm{VO}_{2 \text { max }}$ increases after 12 weeks of training were mainly achieved (2/3) through changes in $\mathrm{Q}_{\max }$ in the young and elderly [16]. Improved left ventricular function accompanied by increases in $\mathrm{VO}_{2 \text { max }}, \mathrm{Q}_{\max }$, and submaximal exercise performance has been shown to occur already after short-term (6 days) exercise training [17]. The authors suggest that these short-term effects are due to the exercise-induced rapid expansion of plasma volume producing enhanced SV and ventricular filling secondary to a Frank-Starling effect. These changes might support cardiac structural adaptations during subsequent long-term training provided training stimuli are sufficiently intense. Conversely, when trained and untrained subjects were exposed to physical inactivity (12 week), $\mathrm{SV}_{\max }$ and $\mathrm{Q}_{\max }$ declined in both groups to a similar extent in the early period of inactivity followed by a later decline in maximal a- $\mathrm{vO}_{2}$ difference [18]. Muscle capillarization and oxidative enzyme activity in the trained, however, remained above sedentary levels explaining why the a- $\mathrm{vO}_{2}$ difference and $\mathrm{VO}_{2}$ max after 12 weeks of inactivity were still higher in trained than in untrained subjects. $\mathrm{Q}_{\max }$ and maximal $\mathrm{DO}_{2}$ rapidly decline as a consequence of coordinated deadaptation to physical inactivity and will markedly improve with regular physical activity, but increases may be especially pronounced when stimuli to the cardiovascular system are sufficiently intense (fig. 2a). Also important for the elderly, prior endurance training helps maintain oxidative capacity of locomotor muscles during a subsequent prolonged period of physical inactivity, which enables the individual to start at higher training intensities resulting in more rapid improvements of $\mathrm{Q}_{\max }$ and $\mathrm{VO}_{2 \text { max }}$.

\section{Oxygen Carrying Capacity}

The oxygen carrying capacity depends on the amount of $\mathrm{Hb}$ of the blood. Most of the oxygen (>98\%) is transported attached to $\mathrm{Hb}$ and changes in $\mathrm{Hb}$ are accompanied by changes in $\mathrm{VO}_{2}$ max. Well-designed studies evaluating the effects of blood doping or those elevating $\mathrm{Hb}$ mass by erythropoietin administration have convincingly demonstrated increases in $\mathrm{VO}_{2 \max }$ by 4-9\% [19]. On the other hand, low $\mathrm{Hb}$ values in anemia are associated with reduced aerobic capacity. Blood-carrying capacity is generally lower in females than males and there is a small decrease with age (approx. 10\% from $30-80$ years) at least in males, which may also contribute to the age-related decline in $\mathrm{VO}_{2}$ max . 


\section{The Vascular System}

Advancing age is typically associated with macrovascular and microvascular dysfunction resulting in stiffening of central elastic arteries, elevation of pressure from wave reflections, and peripheral endothelial dysfunction. These changes also negatively affect blood flow and oxygen delivery to exercising muscles. Nitric oxide (NO), released by endothelial cells, is largely involved in regulating vascular tonus. NO-bioavailability is reduced with aging and might therefore contribute to endothelial dysfunction. On the other hand, regular exercise increases NO-bioavailability and has been suggested as the single most important modulatory influence on vascular aging [20].

\section{Mitochondrial Respiration Capacity of Skeletal Muscles}

The oxidative capacity of the mitochondria of skeletal muscles has been estimated to be at least two times higher than that used during whole body exercise [11], and is unlikely to be a limiting factor to $\mathrm{VO}_{2}$ max in humans. Training-induced large elevations of mitochondrial density and enzymes resulted in only modest $\mathrm{VO}_{2}$ max improvements, indicating that these adaptations are not of limiting importance [21]. The same is true for trainingrelated increases in capillary density which elongate mean transit time, thereby maintaining or improving oxygen extraction [21]. During heavy exercise, almost all of the available oxygen (approx. $200 \mathrm{ml} \mathrm{O}_{2} / \mathrm{l}$ ) is extracted out of the arterial blood, with only about $20 \mathrm{ml} \mathrm{O}_{2} / \mathrm{l}$ in the venous blood draining the exercising muscles. At least in trained males and females, the a- $\mathrm{vO}_{2}$ difference only contributed a little to $\mathrm{VO}_{2 \max }$ reductions with aging [12]. It declines by about $12 \%$ in the untrained elderly and only by $3-5 \%$ in trained old men and women compared to the untrained. A recent study demonstrated that relative $\mathrm{VO}_{2}$ max $(\mathrm{ml} / \mathrm{min} / \mathrm{kg})$ was $46 \%$ higher in long-term endurance-trained older subjects (approx. 71 years) compared to untrained (approx. 71 years) [22]. Time to exhaustion was 35\% longer and capillarization (number of capillaries per fiber) was $27 \%$ higher in the trained subjects compared to the untrained. The authors concluded that metabolic and angiogenic adaptability of skeletal muscle is maintained in elderly subjects at least up to the age of 75 years [22]. Mitochondrial respiration capacity of skeletal muscles varies, dependent on exercise training, over a wide range and is unlikely to be a limiting fac-

Exercise Limitations by the Oxygen

Delivery and Utilization Systems tor to $\mathrm{VO}_{2}$ max even in the untrained elder, but is considered to be of utmost importance to perform sustained exercise.

\section{Capability to Perform Sustained Exercise}

Whereas $\mathrm{VO}_{2 \max }$ can be maintained for no longer than a few minutes, endurance sports are typically performed for a prolonged duration up to several hours. This is also true regarding daily physical activities and leisure time activities of the elderly. Depending on the training state and the exercise duration, only a certain percentage of $\mathrm{VO}_{2}$ max can be used. The highest percentage of $\mathrm{VO}_{2}$ max that can be maintained, e.g. for an hour, is a measure of the capability to perform sustained exercise or of the anaerobic threshold of an individual. Åstrand and Rhodal [23] reported that well-trained subjects could maintain $\mathrm{VO}_{2}$ at 87 and $83 \%$ of $\mathrm{VO}_{2 \text { max }}$ for 1 and $2 \mathrm{~h}$ compared to 50 and $35 \%$ in their untrained peers. Thus, $\mathrm{VO}_{2}$ max does not determine the ability to perform sustained exercise, but represents an upper limit. The ability to use a high percentage of the individual $\mathrm{VO}_{2}$ max results predominantly from chronic adaptations in skeletal muscle due to cumulative effects of repeated bouts of exercise. These adaptations include the increase of key enzyme activities of the mitochondrial electron transport chain and an associated increase in mitochondrial protein accumulation and an increased capillary supply. The resulting improvements in endurance capacity are mainly due to a higher rate of fat oxidation and a concomitant reduction in glycolytic flux, and a tighter control of the acid-base status. Thus, exercise at the same work rate elicits smaller disturbances in homeostasis in the trained muscles, lesser use of muscle glycogen, and decreased lactate production. Consequently, muscle-metaboreceptor activation is reduced, eliciting lower cardiovascular and ventilatory responses and improved exercise tolerance not necessarily associated with $\mathrm{VO}_{2 \max }$ increase. A longitudinal study (10 years) including 441 older men and women aged 55-85 years found no significant decrease in the anaerobic threshold as a percentage of $\mathrm{VO}_{2 \text { max }}$ in men and women, confirming that the anaerobic threshold declines with aging at a slower rate than $\mathrm{VO}_{2}$ max [24]. This may well be related to the observation that the muscle oxidative capacity is affected to a lesser degree by physical activity and inactivity than $\mathrm{Q}_{\max }$, or in other words, changes in maximal $\mathrm{DO}_{2}$ are more rapid and more pronounced compared to those in the a- $\mathrm{vO}_{2}$ difference (oxygen utilization). These observations also indicate that coordinated 
adaptation and deadaptation occur stimuli-dependent differently at the various levels of the lung-heart muscle axis.

\section{Determinants of $\mathrm{Vo}_{2}$ max in Elderly with Cardiopulmonary Diseases}

The prevalence of cardiorespiratory diseases is steeply increasing with age. In patients suffering from lung and/or cardiovascular diseases, ventilatory restriction and/or the pumping function of the heart may become predominantly limiting factors. Mechanisms of coordinated deadaptation and adaptation may differ in some ways from those in healthy subjects and their understanding may help to avoid detrimental effects of deadaptation and to optimize applications of exercise training.

\section{Chronic Obstructive Pulmonary Disease}

Chronic obstructive pulmonary disease (COPD) is characterized by progressive airflow limitation associated with the development of secondary systemic manifestations including respiratory and peripheral muscle dysfunction. The prevalence of COPD according to the Global Initiative on Obstructive Lung Disease (GOLD) stage 2 or higher amounts to $9-10 \%$ in adults aged 40 years and older, smoking being the most important risk factor. Although reduced $\mathrm{FEV}_{1}$ represents an important diagnostic criterion, it is only weakly correlated with $\mathrm{VO}_{2}$ max. Exercise intolerance often represents the most striking symptom in these patients. Beside ventilatory limitations, leg fatigue has been suggested to contribute to exercise intolerance in COPD. Conversely, exercise training evidently improves exercise tolerance [25]. However, limitations to $\mathrm{VO}_{2}$ max at maximal exercise and improvements of $\mathrm{VO}_{2 \text { max }}$ due to exercise training have been discussed controversially and seem mainly to depend on the contribution of limiting factors arising from dysfunction of various organ systems. Plankeel et al. [25] demonstrated that $\mathrm{VO}_{2}$ max increased after exercise training by $4.8 \%$ in patients who were primarily ventilatory limited, by $6.6 \%$ in those who were ventilatory and cardiovascular limited, by $13.2 \%$ in those who were solely cardiovascular limited, and by $15.9 \%$ in those without limitations. In addition, initial average $\mathrm{VO}_{2}$ max values are low in COPD patients, ranging from 0.5 to $1.6 \mathrm{l} / \mathrm{min}$ in most exercise tolerance studies. These findings suggest rapid deadapta- tion of skeletal muscles and cardiovascular function subsequent to ventilatory limitation (fig. $2 b$ ) and the occurrence of only small training adaptations with regard to $\mathrm{DO}_{2 \text { max }}$ and $\mathrm{VO}_{2 \text { max }}$. Minute ventilation (VE) during exercise increases as a consequence of the metabolic demands and to compensate for the developing lactic acidosis above the anaerobic threshold. On the one hand exercise VE in COPD is enhanced due to a larger functional dead space and ventilation-perfusing mismatch, and on the other hand peak VE or ventilatory capacity is reduced, likely explaining ventilatory limitation in these patients. Additionally, dynamic hyperinflation is a common observation in COPD patients, which is well correlated with exercise intolerance and may even occur in aging athletes without COPD [26]. Severe dyspnea and the highly elevated work of breathing may inhibit muscle fiber recruitment and blood flow to locomotor muscles at maximal exercise [27]. The diminished $\mathrm{Q}_{\text {peak }}$ observed in COPD is likely a consequence of the low attainable work rate. It may be assumed that in COPD patients exercising near the upper limit of oxygen transport imposed by impaired lung function, any enhancement of oxygen transport capacity by further increments of cardiac output and/or oxygen extraction would be largely offset by increasing desaturation of the arterial blood. The circulus vitiosus in COPD patients is characterized by low exercise tolerance promoting physical inactivity and worsening of locomotor and respiratory muscle function, contributing to further exercise intolerance (fig. 2b). This represents an impressive example of coordinated deadaptation initiated by the diseased respiratory system followed by negatively affecting the cardiovascular and skeletal muscle systems. Progressive dysfunction of locomotor muscles is associated with anaerobic energy metabolism and lactic acid production. The related enhancement of muscle metaboreflexes contribute to increasing ventilatory requirements and dyspnea responsible for the rapid development of particularly low exercise tolerance in COPD patients. Thus, the prevention or therapy of muscle dysfunction is of utmost importance. One-leg high-intensity interval cycling has been demonstrated to be superior to two legs in improving exercise tolerance and $\mathrm{VO}_{2}$ peak. In addition, pharmacological interventions improving ventilation and respiratory muscle training will improve ventilatory constraints and thus enable higher training intensities and skeletal muscle and cardiovascular adaptations, resulting in more pronounced increases in $\mathrm{Q}_{\max }$ and $\mathrm{VO}_{2}$ max . 


\section{Chronic Heart Failure}

The prevalence of chronic heart failure (CHF) increases with age and rises from about $1.5 \%$ at the age of 40 to almost $15 \%$ in those aged 80 years or above. Myocardial infarction is the leading cause for the development of CHF and the incidence of both myocardial infarction and CHF increases dramatically with age. Heart failure develops along with left ventricular remodeling, i.e. left ventricular dilation, hypertrophy, fibrosis, and infarct expansion, resulting in a dilated and poorly functioning ventricle. CHF is associated with a high mortality rate and a poor quality of life, particularly due to the low exercise tolerance and related fatigue and breathlessness. The inadequate increase in cardiac output and the related reduction in muscle blood flow during exercise may be considered as a main reason for the initiation of the deadaptation of skeletal muscles (fig. 2c). Esposito et al. [28] demonstrated by adopting 1-leg and 2-leg exercise and elevating $\mathrm{CaO}_{2}$ by administration of hyperoxia that cardiac output and oxygen delivery significantly contributed to exercise limitation. The elegant experimental design elicited both the muscle convective component, defined by the Fick principle $\left[\mathrm{VO}_{2}=\right.$ blood flow $\left.\left(\mathrm{CaO}_{2}-\mathrm{CvO}_{2}\right)\right]$, and the muscle diffusive component $\left(\mathrm{D}_{\mathrm{M}} \mathrm{O}_{2}\right)$, defined by Fick's law of diffusion $\left(\mathrm{VO}_{2}=\mathrm{D}_{\mathrm{M}} \mathrm{O}_{2} \times \mathrm{PvO}_{2}\right)$ to contribute to $\mathrm{VO}_{2 \text { peak }}$ limitation in CHF patients. Morphological, enzymatic, and functional skeletal muscle abnormalities in those patients are strikingly similar to those observed after prolonged immobilization, e.g. due to bed rest. The reduced myocardial functioning initiates physical inactivity followed by deadaptation of locomotor and probably also of respiratory muscles. On the other hand, many studies provide promising results from exercise training in patients suffering from CHF. Depending on the type of training, skeletal muscle function, and probably cardiac function, will improve. Small muscle mass exercises, e.g. knee extensor exercises, which are not stressing the cardiorespiratory system have been shown to induce various peripheral structural and functional adaptations improving $\mathrm{VO}_{2}$ peak without changing cardiac output [29]. Muscle training increased muscle capillarity and mitochondrial density (21 and 25\%) and leg oxygen delivery and diffusive conductance. At similar submaximal exercise intensities, these peripheral adaptations are associated with improved exercise tolerance characterized by diminished production of lactate and lesser leg fatigue accompanied by reduced cardiorespiratory responses, e.g. lower heart rates and VE. Several whole-body training studies demonstrated beneficial effects on skeletal muscle function, reversal of left ventricular remodeling, $\mathrm{VO}_{2}$ max , exercise performance, and quality of life [30]. Most important, however, highintensity training in particular seems to provoke coordinated adaptations at peripheral and systemic levels, i.e. skeletal muscle adaptations, left ventricular remodeling, and $\mathrm{VO}_{2}$ max increase even in elderly patients with $\mathrm{CHF}$ and severely impaired cardiovascular function [30]. These observations are in line with the concept that adaptations are coordinated at the level where stimuli are acting, i.e. peripherally or peripherally and systemically.

\section{General Training Recommendations}

The maintenance or the attainment of high aerobic exercise capacity and exercise tolerance are important training goals for aging individuals. Healthy elderly after prolonged detraining periods or those with cardiorespiratory diseases may especially benefit from starting with training of small muscle groups, e.g. one-leg or arm exercises including resistance training, primarily resulting in improved skeletal muscle function and exercise tolerance. Subsequently, individuals will better tolerate whole-body exercises, e.g. walking, running, cycling, and swimming at intensities challenging the cardiorespiratory system, particularly favoring cardiovascular adaptations and $\mathrm{VO}_{2 \text { max }}$ increases. High-intensity interval training seems often to be superior to low- or moderate-intensity continuous training.

\section{Conclusion}

Increasing physical inactivity in aging persons initiates a circulus vitiosus resulting in coordinated deadaptation of the oxygen delivery and utilization systems mainly affecting the heart-muscle axis. Whereas in the healthy elderly the circulus vitiosus starts from inactive locomotor muscles, the lung or the heart represent the origin in patients suffering from respiratory or cardiovascular diseases. Specific exercise training programs, considering the state of cardiorespiratory health and physical activity, are the most important and almost the only effective intervention to avoid or to break the circulus vitiosus, thereby promoting quality and expectancy of live in aging humans.

\section{Disclosure Statement}

There is no conflict of interest. 


\section{References}

1 Kokkinos P, Sheriff H, Kheirbek R: Physical inactivity and mortality risk. Cardiol Res Pract 2011;2011:924945.

-2 Burtscher M, Förster H, Burtscher J: Superior endurance performance in aging mountain runners. Gerontology 2008;54:268-271.

$\checkmark 3$ Weibel ER, Taylor CR, Hoppeler H: The concept of symmorphosis: a testable hypothesis of structure-function relationship. Proc Natl Acad Sci USA 1991;88:10357-10361.

4 Hill AV, Lupton H: Muscular exercise, lactic acid, and the supply and utilization of oxygen. Q J Med 1923;16:135-171.

$\checkmark 5$ Burtscher M, Nachbauer W, Wilber R: The upper limit of aerobic power in humans. Eur J Appl Physiol 2011;111:2625-2628.

$\checkmark 6$ Hsia CC: Coordinated adaptation of oxygen transport in cardiopulmonary disease. Cir culation 2001;104:963-969.

-7 Amann M: Pulmonary system limitations to endurance exercise performance in humans. Exp Physiol 2012;97:311-318.

$>8$ Taylor BJ, Johnson BD: The pulmonary circulation and exercise responses in the elderly. Semin Respir Crit Care Med 2010;31:528538.

-9 Habedank D, Reindl I, Vietzke G, Bauer U, Sperfeld A, Gläser S, Wernecke KD, Kleber FX: Ventilatory efficiency and exercise tolerance in 101 healthy volunteers. Eur J Appl Physiol Occup Physiol 1998;77:421-426.

10 McClaran SR, Babcock MA, Pegelow DF, Reddan WG, Dempsey JA: Longitudinal effects of aging on lung function at rest and exercise in healthy active fit elderly adults. J Appl Physiol 1995;78:1957-1968.

11 Saltin B: Hemodynamic adaptations to exercise. Am J Cardiol 1985;55:42D-47D.

-12 Ogawa T, Spina RJ, Martin WH 3rd, Kohrt WM, Schechtman KB, Holloszy JO, Ehsani AA: Effects of aging, sex, and physical training on cardiovascular responses to exercise. Circulation 1992;86:494-503.
13 Sagiv M, Goldhammer E, Ben-Sira D, Amir R: What maintains energy supply at peak aerobic exercise in trained and untrained older men? Gerontology 2007;53:357-361.

14 Pimentel AE, Gentile CL, Tanaka H, Seals DR, Gates PE: Greater rate of decline in maximal aerobic capacity with age in endurancetrained vs. sedentary men. J Appl Physiol 2003;94:2406-2413.

15 Tanaka H, Monahan KD, Seals DR: Age-predicted maximal heart rate revisited. J Am Coll Cardiol 2001;37:153-156.

16 Murias JM, Kowalchuk JM, Paterson DH: Time course and mechanisms of adaptations in cardiorespiratory fitness with endurance training in older and young men. J Appl Physiol 2010;108:621-627.

17 Goodman JM, Liu PP, Green HJ: Left ventricular adaptations following short-term endurance training. J Appl Physiol 2005;98: 454-460.

18 Coyle EF, Martin WH 3rd, Sinacore DR, Joyner MJ, Hagberg JM, Holloszy JO: Time course of loss of adaptations after stopping prolonged intense endurance training. J Appl Physiol 1984;57:1857-1864.

19 Gledhill N: The influence of altered blood volume and oxygen transport capacity on aerobic performance. Exerc Sport Sci Rev 1985; 13:75-93.

20 Seals DR, Moreau KL, Gates PE, Eskurza I: Modulatory influences on ageing of the vasculature in healthy humans. Exp Gerontol 2006;41:501-507.

21 Saltin B, Henriksson J, Nygaard E, Andersen P: Fiber types and metabolic potentials of skeletal muscles in sedentary man and endurance runners. Ann NY Acad Sci 1977; 301:3-29.

22 Iversen N, Krustrup P, Rasmussen HN, Rasmussen UF, Saltin B, Pilegaard H: Mitochondrial biogenesis and angiogenesis in skeletal muscle of the elderly. Exp Gerontol 2011;46: 670-678.
23 Åstrand PO, Rodahl K: Textbook of Work Physiology. New York, McGraw-Hill, 1970, pp 279-430.

24 Stathokostas L, Jacob-Johnson S, Petrella RJ, Paterson DH: Longitudinal changes in aerobic power in older men and women. J Appl Physiol 2004;97:781-789.

25 Plankeel JF, McMullen B, MacIntyre NR: Exercise outcomes after pulmonary rehabilitation depend on the initial mechanism of exercise limitation among non-oxygen-dependent COPD patients. Chest 2005;127: 110-116.

26 Burtscher M, Schocke M, Koch R: Ventilation-limited exercise capacity in a 59-yearold athlete. Respir Physiol Neurobiol 2011; 175:181-184.

27 Harms CA, Babcock MA, McClaran SR, Pegelow DF, Nickele GA, Nelson WB, Dempsey JA: Respiratory muscle work compromises leg blood flow during maximal exercise. J Appl Physiol 1997;82:1573-1583.

28 Esposito F, Mathieu-Costello O, Shabetai R, Wagner PD, Richardson RS: Limited maximal exercise capacity in patients with chronic heart failure: partitioning the contributors. J Am Coll Cardiol 2010;55:1945-1954.

29 Esposito F, Reese V, Shabetai R, Wagner PD, Richardson RS: Isolated quadriceps training increases maximal exercise capacity in chronic heart failure: the role of skeletal muscle convective and diffusive oxygen transport. J Am Coll Cardiol 2011;58:13531362.

30 Wisløff U, Støylen A, Loennechen JP, Bruvold M, Rognmo Ø, Haram PM, Tjønna AE, Helgerud J, Slørdahl SA, Lee SJ, Videm V, Bye A, Smith GL, Najjar SM, Ellingsen $\varnothing$, Skjaerpe T: Superior cardiovascular effect of aerobic interval training versus moderate continuous training in heart failure patients: a randomized study. Circulation 2007;115: 3086-3094. 